\title{
Searches for supersymmetry in resonance production, R-parity violating signatures and events with long-lived particles at LHC
}

\author{
Sofia VALLECORSA* \\ On behalf of the ATLAS and CMS collaborations \\ University of Geneva \\ E-mail: sofia.vallecorsaecern.ch
}

\begin{abstract}
An extended QCD sector beyond the minimal supersymmetric standard model or the admission of R-parity violation introduces new signatures to the search for supersymmetry at the LHC. Strongly interacting resonances may decay to jets, sleptons via lepton-flavour violating processes and lightest supersymmetric particles may decay into many leptons with or without missing transverse momentum. Several supersymmetric models also predict massive long-lived supersymmetric particles. Such particles may be detected through abnormal specific energy loss, appearing or disappearing tracks, displaced vertices, long time-of-flight or late calorimetric energy deposits. These proceedings present recent results from searches for supersymmetry in resonance production, R-parity violating signatures and events with long-lived particles with the ATLAS and CMS detectors.
\end{abstract}

XXII. International Workshop on Deep-Inelastic Scattering and Related Subjects 28 April - 2 May 2014

Warsaw, Poland

\footnotetext{
* Speaker.
} 


\section{Introduction}

An essential aspect of all supersymmetric (SUSY) models is the R-parity quantum number $R=(-1)^{3 B+L+2 S}$, where $B$ denotes the baryon number, $L$ the lepton number and $S$ the spin of a particle. The conservation of $R$-parity leaves the lightest supersymmetric particle stable and makes it a natural dark matter candidate. However, in the most general formulations of supersymmetry $R$-parity is not conserved, allowing couplings between ordinary fermions and squarks or sleptons. When $R$-parity violating terms $\left(W_{R P V}\right)$ are introduced into the superpotential, the Lightest Supersymmetric Particle (LSP) can decay into Standard Model (SM) particles. $W_{R P V}$ can be written as [1]:

$$
W_{R P V}=\lambda_{i j k} L_{i} L_{j} \bar{E}_{k}+\lambda_{i j k}^{\prime} L_{i} Q_{j} \bar{D}_{k}+k_{i} L_{i} H_{2}+\lambda_{i j k}^{\prime \prime} \bar{D}_{i} \bar{D}_{j} \bar{D}_{k}
$$

where $L_{i}$ and $Q_{i}$ denote the lepton and quark SU(2) doublet superfields, respectively, while the corresponding singlet are given by $\bar{E}_{i}$ and $\bar{D}_{i}$. $H_{2}$ denotes the Higgs SU(2) doublet that couples to up-like quarks. $\lambda_{i j k}, \lambda_{i j k}^{\prime}$ and $\lambda_{i j k}^{\prime \prime}$ are new Yukawa couplings and the $k_{i}$ have dimensions of mass, but vanish at the unification scale. The indices $i, j$ and $k$ refer to quark and lepton generations. Terms with $\lambda_{i j k}$ or $\lambda_{i j k}^{\prime}$ different than zero represent lepton violating couplings, $\lambda_{i j k}^{\prime \prime}$ generate baryon number violation: the stability of the proton forbids simultaneous lepton and baryon number violation. This paper will look at the latest results in the search for R-parity violating (RPV) SUSY in multi-leptonic and multi-jet final states. The ATLAS [2] and CMS [3] collaborations have produced several results within this scope: here, they are grouped according to the final state and the terms in the RPV superpotential they probe. The second part of these proceedings is dedicated to long-lived particle events. If $\lambda_{i j k}, \lambda_{i j k}^{\prime}$ or $\lambda_{i j k}^{\prime \prime}$ are small, the LSP can have a long lifetime and decay far away from the interaction point, generating largely displaced vertices, or even cross the whole detector before decaying. It should be noted, however, that some R-parity conserving scenarios can also give rise to long-lifetime signatures: for example, if the difference between the mass of the next-to-lightest SUSY particle (NLSP) and the LSP is small.

All the analyses described here use data collected at a center-of-mass energy of 7 or $8 \mathrm{TeV}$, in 2011-2012 with the ATLAS and CMS detectors at the LHC [7-19]. The analysed data correspond to a total integrated luminosity of about $20 \mathrm{fb}^{-1}$ in most cases.

\section{R-Parity Violating searches: massive particles to multi-jets and multi-leptons final states}

R-parity violating scenarios do not exhibit the typical signature of most SUSY searches: since the LSP can decay to SM particles, a large missing energy signature is not to be expected and searches must rely on different signatures such as a large number of jets and/or leptons in the final state. Decays to multi-jets are mediated by the baryon violating term $\lambda_{i j k}^{\prime \prime} \bar{D}_{i} \bar{D}_{j} \bar{D}_{k}$ including one up-type and two down-type quarks. Therefore the jet flavour information can be used to constrain different $\lambda$ "'s. This kind of searches use rather simple strategies: the jet multiplicity, jet $p_{T}$ and the number of heavy flavour jets are used to separate the signal from the background, which is represented mostly by QCD multiple jet production. On the other hand, when heavy flavour jets are considered, $t \bar{t}$ and $W+$ jet become the dominant contribution. The total background can be estimated from data selecting low jet multiplicity control regions and projecting the result to 

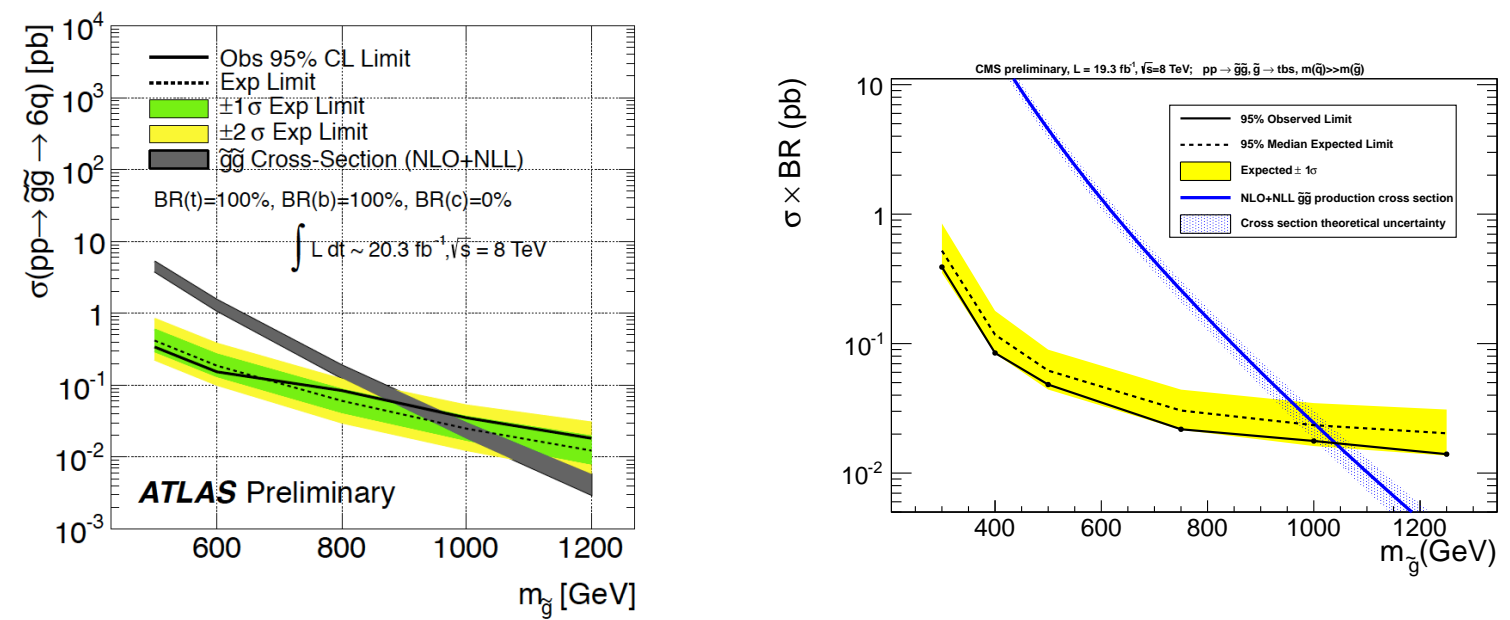

Figure 1: (Left) ATLAS: expected and observed cross-section limits for the 6-quark gluino models for the case where gluinos decay into a $t$ and $b$-quark in the final state. [7]. (Right) CMS: 95\% C.L. limit on gluino pair production cross section. The signal considered is $p p \rightarrow \tilde{g} \tilde{g}$ followed by the decay $\tilde{g} \rightarrow t b s$ [9].

higher multiplicity regions. ATLAS has published results of a search optimised on two signal regions corresponding to different gluino decays: direct RPV gluinos, decaying to three quarks, or gluinos decaying to two quarks plus neutralino, followed by RPV neutralino decays to three quarks. Considering that gluinos are produced in pairs, the two scenarios give rise to final states including six or ten jets respectively. No excess with respect to Standard Model is observed and limits are categorised according to the gluinos to heavy quarks branching ratios. Fig. 1 shows the limit corresponding to the six jet case for gluinos decaying to heavy flavour: gluinos with masses up to $900-1000 \mathrm{GeV}$ are excluded. A similar result is obtained for the ten jets events (details can be found in [7]). CMS has shown results for two different analyses: one targeted to RPV gluinos decaying to three quarks final states [8], similar to the ATLAS case, and a more specific search aimed at reconstructing the $\tilde{g} \rightarrow t b s$ decay [9]. The first analysis requires at least 3 light flavour jets in the event or two light flavour and one heavy flavour jet. For each event, jet triplets are formed and their invariant mass is calculated to separate the signal from the background (mostly QCD production, $t \bar{t}$ and combinatorial background). Decays with at least one heavy flavour are excluded for gluino masses up to $835 \mathrm{GeV}$, while decays to light quarks are excluded up to $650 \mathrm{GeV}$. The second analysis, optimised for a specific decay channel uses heavy jets multiplicity to separate signal from background. It combines results for three signal regions including six, seven or at least eight b-tagged jets in the final state and it excludes gluino masses up to more than $1 \mathrm{TeV}$ [9]. The exclusion limit is shown on the right panel in Fig.1.

When probing the lepton violating term, $\lambda_{i j k} L_{i} L_{j} \bar{E}_{k}+\lambda_{i j k}^{\prime} L_{i} Q_{j} \bar{D}_{k}$ one can expect a large number of leptons in the final state. Typical searches can reach high signal to background ratios simply relying on inclusive single or double lepton triggers and basic selection of isolated high $p_{T}$ leptons in the final state. A study of the di-lepton invariant mass is also needed in order to separate the events associated to $Z$ boson production. Backgrounds can be classified as irreducible, when events include several real leptons originating from Standard Model processes such as heavy boson production: $t \bar{t} H, W H$, di-bosons, with or without jets, $W W Z$ and $W Z Z$ or $Z Z \bar{t}$. Their contribution 

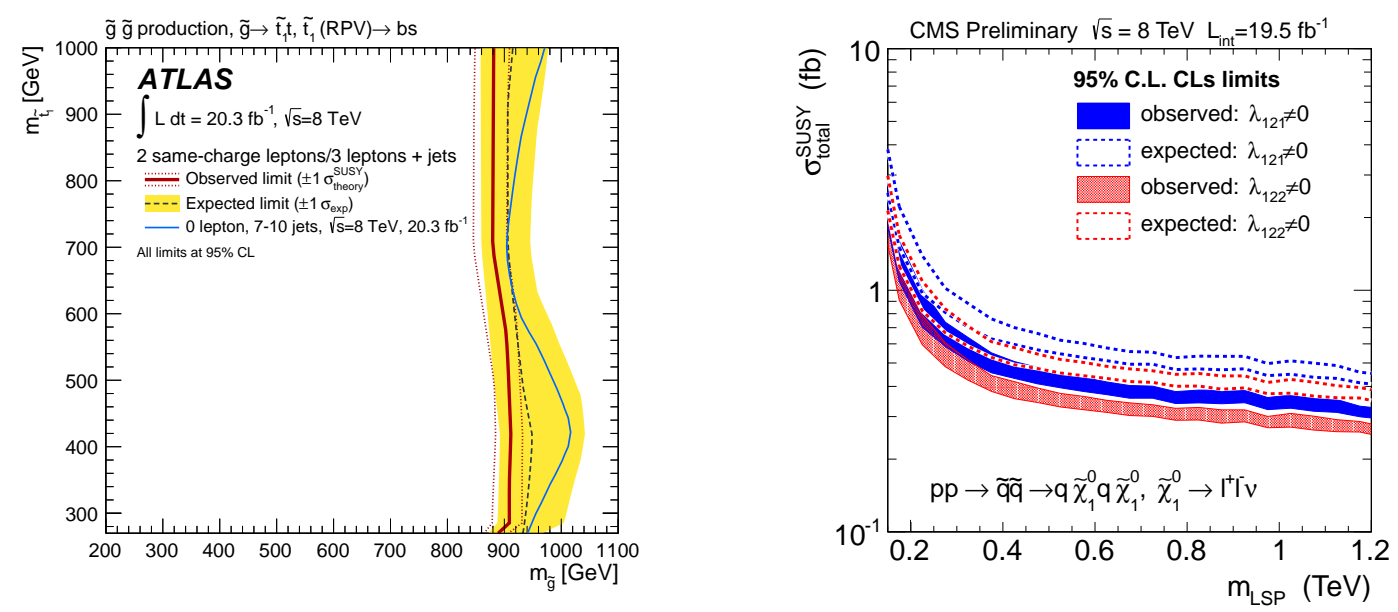

Figure 2: (Left) ATLAS:Observed and expected exclusion limits on gluino-mediated top squark production, obtained with $20.3 \mathrm{fb}^{-1}$ of pp collisions at $\sqrt{s}=8 \mathrm{TeV}$. (Right) CMS: 95\% C.L. upper limit on total cross section, for generic SUSY models, for the lepton flavors mixture corresponding to $\lambda_{121} \neq 0$ and $\lambda_{122} \neq 0$.

is studied using montecarlo simulated samples. On the other hand, reducible background originates from cases where at least one of the leptons in the event is fake: typically a jet or a photon misidentified as a lepton. Reducible background can be estimated directly from the data. Both ATLAS and CMS published exclusion limits for multi-lepton searches: NLSP gluinos are excluded up to masses of $1-1.4 \mathrm{TeV}$, RPV stop decays up to $1 \mathrm{TeV}$ stop masses; LSP neutralino exclusion ranges between 400 and $800 \mathrm{GeV}$. As an example, Fig. 2 shows the ATLAS result for RPV decaying top squark [12], on the left panel, and the CMS limit [10] on neutralino mediated gluino decays, on the right.

\section{Long-lived particle searches}

With or without R-parity conservation, searches for events with long-lived particles don't rely on missing transverse energy. In RPV scenarios the lifetime of the LSP is proportional to $\lambda^{-2}$ and typical decay lengths can span several orders of magnitudes ranging from a few millimetres (a displaced decay vertex can be reconstructed) up to several meters, in which case the long-lived particle will be stable on the detector scale and can be identified as a massive, highly penetrating slow particle, with large momentum, high ionisation energy loss and large time of flight. R-parity conserving scenarios can also give rise to long-lived particle signatures: in SplitSUSY [4], gluinos acquire a long lifetime because their decay is mediated by heavy squarks: in this scenario, RHadrons, gluino-quark bound states, are formed. In some Gauge-Mediated Supersymmetry Breaking (GMSB [5]) models the weak coupling between the NLSP and the gravitino can generate stable leptons or delayed photons. In the case of stable massive particles, both lepton-like or coloured, one can expect striking detector signatures that might require specific online selection and identification techniques. For example, the ATLAS long-lived slepton search [13] relies on a muon reconstruction algorithm, modified in order to efficiently reconstruct slow moving particles in the muon spectrometer. It is able to estimate the speed of a particle with a resolution of about $2 \%$ down to very low $\beta$ values $(\beta \approx 0.4$ ). Using this technique, stable massive leptons could be excluded 

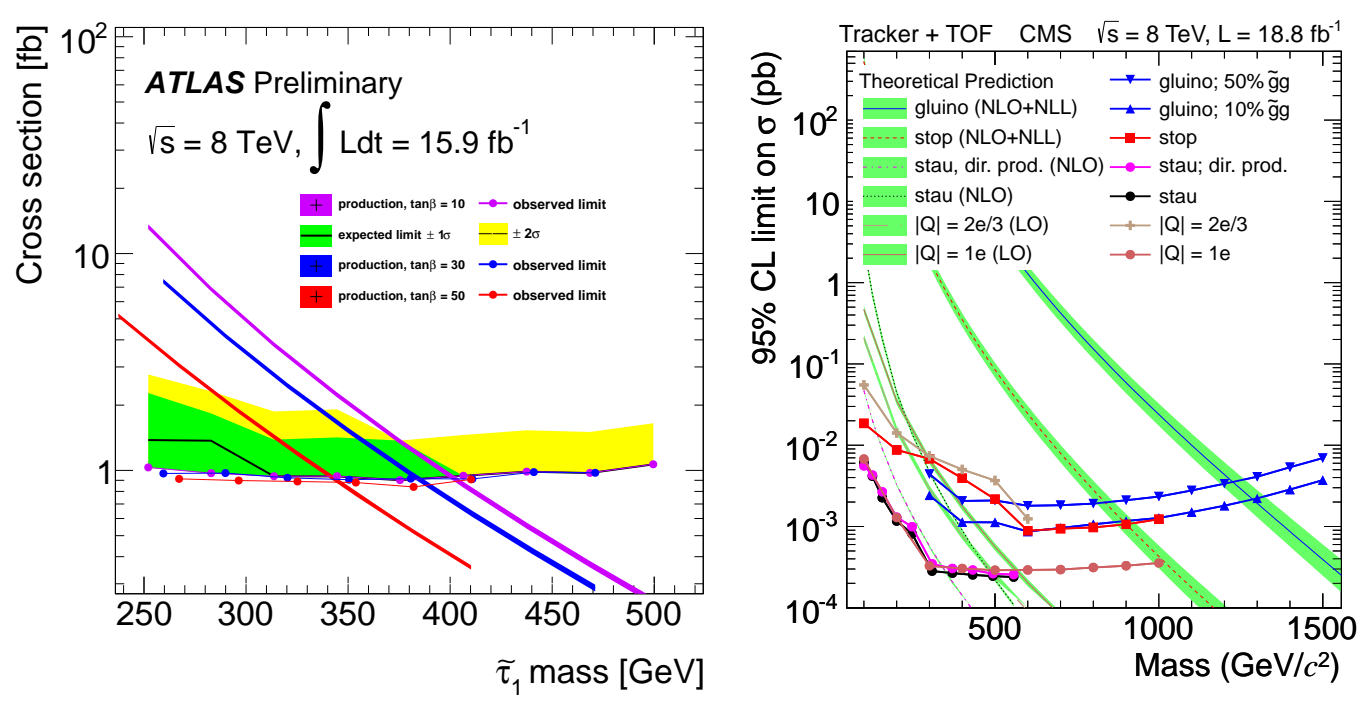

Figure 3: (Left) ATLAS: Cross-section limits as a function of the $\tilde{\tau}_{1}$ mass in GMSB models. Observed limits are given as solid lines with markers. The theoretical prediction for the cross-section is shown as a colored $1 \sigma$ band. Different colours represent models with different $\tan \beta$. Expected limits for $\tan \beta=10$ are drawn as black lines with \pm 1 and $\pm 2 \sigma$ uncertainty bands drawn in green and yellow respectively. (Right) CMS: Cross section upper limits at 95\% C.L. on various signal models for the tracker+TOF analysis for 2012 data (at $8 \mathrm{TeV})$.

up to 350-400 GeV masses [13]. A similar CMS search for R-Hadrons excludes stable stops and gluinos up to masses of $900 \mathrm{GeV}$ and 1.2-1.3 TeV respectively [14] (see figure 3).

A special case is represented by long-lived gluinos that stop in the detector before decaying. A specific search presented by the ATLAS collaboration excludes such gluinos up to $800 \mathrm{GeV}$ for lifetimes up to $1000 \mathrm{sec}$ and $600 \mathrm{GeV}$ for lifetimes up to 2 years [15].

For intermediate decay lengths, when the decay happens inside the detector it is possible to reconstruct a displaced decay vertex from high $p_{T}$ leptons or jets. This kind of searches have the advantage of featuring relatively low background, mostly due to cosmic rays, random combinations of tracks or hadronic interactions with material. On the other hand, standard reconstruction algorithms, optimised to select events centred on the primary interaction vertex, have low efficiencies $(<30 \%)$ that rapidly decrease with the decay vertex distance from the primary vertex. Both ATLAS and CMS have results for searches including one or more leptons (see [16] and [17]) or jets [18].

Another interesting signature is given by some Anomaly Mediated Supersymmetry Breaking models (AMSB [6]) that predict a difference between the chargino and neutralino masses of about $160 \mathrm{MeV}$ : in these scenarios, charginos decaying to neutralinos plus pions look like disappearing or "kinked" tracks inside the detector. The main source of background is then due to the misidentification of leptons, the wrong estimation of particle $p_{T}$ or hadrons interacting in the detector material. This background can be estimated directly from the data, fitting the $p_{T}$ spectrum of the disappearing tracks. ATLAS has published strong limits on AMSB charginos (200 to $500 \mathrm{GeV}$ depending on the lifetime) [19] looking for well reconstructed tracks in the inner silicon tracker (Pixel detector and SCT), which have a low number of hits in the outer tracker (TRT) (see [2] for details on detector descriptions). 


\section{Summary}

These proceedings summarise a selection of recent results on RPV SUSY and long-lived particle searches at a centre of mass energy of 7 and $8 \mathrm{TeV}$, with the ATLAS and CMS detectors at the LHC. LHC experiments probe a large range of scenarios and observe no excess over the SM expectation. Exclusion limits are set on the various models. These searches take advantage of unique detector signatures predicted by the different models and often require non standard analysis techniques that push detectors to their limits.

For most of the models studied here, these limits represent the strongest or even the first available constraints and the RPV sector remains far less constrained than R-parity conserving scenarios. With the new LHC run starting in 2015, at $13-14 \mathrm{TeV}$ centre of mass energy, it will be possible to test a even wider RPV phase space. The interested reader is invited to read the detailed papers on each of these analyses, listed in the bibliography.

\section{References}

[1] S. Weinberg, Phys. Rev. D26 (1982) $287 Đ 302$.

[2] ATLAS Collaboration, JINST(2008) 3 S08003.

[3] CMS Collaboration, JINST 3 (2008) S08004.

[4] N. Arkani-Hamed and S. Dimopoulos, JHEP 06 (2005) 073.

[5] M. Dine and W. Fischler, Phys. Lett. B110 (1982) 227.

[6] G. F. Giudice, M. A. Luty, H. Murayama, and R. Rattazzi, JHEP 12027 (1998), arXiv:hep-ph/9810442.

[7] ATLAS Collaboration, ATLAS-CONF-2013-091, http://cds.cern.ch/record/1595753.

[8] CMS Collaboration, Physics Letters B 730 (2014) 193Đ214.

[9] CMS Collaboration, PAS-SUS-12-015, http://cds.cern.ch/record/1430715.

[10] CMS Collaboration, PAS-SUS-13-010, http://cds.cern.ch/record/1563142.

[11] ATLAS Collaboration, ATLAS-CONF-2013-036, http://cds.cern.ch/record/1532429.

[12] ATLAS Collaboration, arxiv:1404.2500.

[13] ATLAS Collaboration, ATLAS-CONF-2013-058, http://cds.cern.ch/record/1557775.

[14] CMS Collaboration, JHEP 07(2013)122.

[15] ATLAS Collaboration, Phys. Rev. D 88, 112003 (2013).

[16] ATLAS Collaboration, ATLAS-CONF-2013-092, http://cds.cern.ch/record/1595755.

[17] CMS Collaboration, PAS-EXO-12-037, http://cds.cern.ch/record/1669814.

[18] CMS Collaboration, PAS-EXO-12-038, http://cds.cern.ch/record/1563591.

[19] ATLAS Collaboration, Phys. Rev. D 88, 112006 (2013). 